\title{
Evaluating overweight and obesity prevalence in survivors of childhood brain tumors: a systematic review protocol
}

\author{
Kuan-Wen Wang ${ }^{1,2,3}$, Adam Fleming ${ }^{1,4}$, Sheila K. Singh ${ }^{5,6}$, Laura Banfield ${ }^{7}$, Russell J. de Souza ${ }^{8}$,
} Lehana Thabane $e^{8,9,10,11}$ and M. Constantine Samaan ${ }^{1,2,3,8^{*}}$ id

\begin{abstract}
Background: Overweight and obesity are well-known risk factors for cardiometabolic diseases including hypertension, myocardial infarction, stroke, and type 2 diabetes in the general population. Survivors of childhood brain tumors (SCBT) are at risk of premature mortality, and recent evidence suggests that these cardiometabolic diseases are potential emerging determinants of survival and quality of life. Therefore, the rates of overweight and obesity in this population need to be examined to assess their impact on outcomes. The objective of this systematic review is to examine the prevalence of overweight and obesity in SCBT. The secondary aim of this review is to evaluate whether SCBT have higher adiposity compared to the general population.
\end{abstract}

Methods: Searches will be conducted in MEDLINE, CINAHL, EMBASE, Cochrane Database of Systematic Reviews, Cochrane Central Register of Controlled Trials, PubMed, and Database of Abstracts of Reviews of Effect. For gray literature, we will search ProQuest Dissertations and Theses A\&l and Web of Science. Two reviewers will independently screen all articles against predetermined eligibility criteria and complete data abstraction, risk of bias, and quality assessments. The primary outcome includes the prevalence of overweight or obesity. The secondary outcomes involve waist-to-hip ratio, waist-to-height ratio, body fat percentage, and skinfold thickness. Meta-analysis will be performed when two or more studies with similar design, populations, and outcomes are available.

Discussion: This review will summarize current data on the prevalence of overweight and obesity in SCBT. This will help the development of an understanding of the scale of overweight and obesity in this population and guide the design of interventions that will improve outcomes.

Systematic review registration: PROSPERO CRD42016051035

Keywords: Systematic review protocol, Protocol, Obesity, Childhood brain tumor, Cancer survivorship

\section{Background}

Recent advances in the management of pediatric brain tumors have significantly improved survival rates $[1,2]$. However, the new record longevity noted in Survivors of Childhood Brain Tumors (SCBT) is being hindered by the emergence of new comorbidities including cardiometabolic diseases like hypertension, myocardial infarction, stroke, and type 2 diabetes [3-13]. The current

\footnotetext{
*Correspondence: samaanc@mcmaster.ca

'Department of Pediatrics, McMaster University, 1280 Main Street West,

HSC-3A57, Hamilton, Ontario L8S 4K1, Canada

2Division of Pediatric Endocrinology, McMaster Children's Hospital, Hamilton,

Ontario, Canada

Full list of author information is available at the end of the article
}

global overweight and obesity epidemic has been blamed for the rise of these cardiometabolic disorders in the general population, but the scale of overweight and obesity and its role in driving adverse outcomes in survivors is unknown.

Of note, SCBT have several risk factors that predispose them to overweight and obesity. These include impaired satiety signals, lower physical activity, impaired mobility and coordination, pain, disrupted sleep, mental health concerns, pituitary hormonal deficiencies, and medications [14-17]. To further understand the contribution of overweight and obesity to cardiometabolic risk in SCBT, there is a need to determine its scale in SCBT. This will 
inform the design of interventions to target overweight and obesity and their risk factors to improve cardiometabolic outcomes, quality of life, and survival rates in this population.

In this systematic review, the epidemiological data on the prevalence of overweight and obesity in SCBT will be evaluated. The primary aim of this review is to determine whether SCBT have higher rates of overweight or obesity compared to non-cancer counterparts. The secondary aim of this review is to evaluate whether SCBT have higher adiposity compared to the general population.

\section{Methods}

This protocol is developed according to the Preferred Reporting Items for Systematic Review and MetaAnalysis-Protocols (PRISMA-P) statement [18, 19] (Additional file 1).

\section{Literature search}

Searches will be conducted in MEDLINE, CINAHL, EMBASE, Cochrane Database of Systematic Reviews, Cochrane Central Register of Controlled Trials, PubMed, and Database of Abstracts of Reviews of Effect. The following concepts along with their synonyms will be used in the search: pediatric, brain tumors, overweight/obesity, and survivors. A search strategy will be developed in consultation with a senior health sciences librarian with expertise in systematic reviews. We will not set any restrictions on publication date, but will restrict our search to English language publications. A full search strategy for MEDLINE is reported in Table 1.

To identify grey literature, we will search ProQuest Dissertations and Theses A\&I and Web of Science. The search in the latter database will be limited to "Conference Proceedings Citation Index-Science-1990-present." We will then search for relevant publications from the first and last authors of the relevant conference abstracts to identify articles originating from the work presented in the abstracts. The reference lists of eligible studies and relevant reviews will also be searched to identify any additional studies. Searches will be updated to capture recent publications by setting publication date restrictions.

The search results will be de-duplicated in EndNote $\mathrm{X} 7[20]$ and then exported into an excel file to screen for eligible titles and abstracts. The full texts of relevant records will then be retrieved to screen against the eligibility criteria.

\section{Study selection and eligibility criteria}

Two independent reviewers, who will meet after each stage to resolve conflicts and achieve consensus, will screen the title and abstract of each record. A third reviewer will be consulted when disagreements persist. The two reviewers will then independently screen the full text of the relevant studies identified from the title and abstract screening.

This review will include SCBT diagnosed under 18 years of age. The following eligibility criteria will be applied: (1) Primary research articles with observational study design including longitudinal cohort, crosssectional, or case-control studies. (2) Sample size of $\geq 10$ patients as previously described [21]. (3) Assessment of prevalence of overweight or obesity and/or body composition using measures including Body Mass Index (BMI), BMI z-score, BMI percentile, waist-to-hip ratio, waist-to-height ratio, body fat, and skinfold thickness. The screening process and results will be reported in a PRISMA flow diagram, as previously described [22-24] (Fig. 1).

\section{Data collection}

We developed a data abstraction form that will be piloted by two reviewers on two eligible studies. Comments will then be incorporated to finalize the form for this specific systematic review. The abstracted data will include publication information of title, authors' names, journal name, year of publication, as well as the city and country of publication. We will also collect study details including setting, study design, eligibility criteria, sample size, study duration, and funding source. Outcome measures, primary findings, and conclusions will be collected as well.

We will extract survivors' characteristics including age at diagnosis of brain tumor, age at study enrollment, and sex. We will also extract brain tumor details including brain tumor type and location and treatment details such as treatment period, duration since treatment completion, and types of treatments received including radiotherapy, chemotherapy, and surgery or combination therapies with these modalities. If the study has a noncancer comparison group, we will document the type and source of non-cancer controls used and abstract the same data except for tumor- and treatment-related variables.

Two reviewers will perform data abstraction independently, followed by a discussion to resolve discrepancies. A third reviewer will intervene to resolve persisting differences. In studies that report the data from multiple cancer types as aggregates, data specific to the brain tumor group will be extracted either through published subgroup data or by contacting the research team to acquire the data. We will also contact the corresponding authors of a published work in attempts to obtain any missing data.

The primary outcome for this review is the prevalence of overweight or obesity estimated by BMI, BMI z-score, 
Table 1 Search strategy for MEDLINE

\begin{tabular}{|c|c|}
\hline$\#$ & Searches \\
\hline 1 & $\exp$ Child/ \\
\hline 2 & child*.ab,ti,kf. \\
\hline 3 & p?ediatric*.ab,ti,kf. \\
\hline 4 & exp Adolescent/ \\
\hline 5 & adolescen*.ab,ti,kf. \\
\hline 6 & youth*.ab,ti,kf. \\
\hline 7 & teen*.ab,ti,kf. \\
\hline 8 & kid*.ab,ti,kf. \\
\hline 9 & 1 or 2 or 3 or 4 or 5 or 6 or 7 or 8 \\
\hline 10 & exp Brain Neoplasms/ \\
\hline 11 & exp Neuroectodermal Tumors/ \\
\hline 12 & exp Glioma/ \\
\hline 13 & glioma*.ab,ti,kf. \\
\hline 14 & astrocytoma*.ab,ti,kf. \\
\hline 15 & oligoastrocytoma*.ab,ti,kf. \\
\hline 16 & astroglioma*.ab,ti,kf. \\
\hline 17 & glioblastoma*.ab,ti,kf. \\
\hline 18 & craniopharyngioma*.ab,ti,kf. \\
\hline 19 & ependymoma*.ab,ti,kf. \\
\hline 20 & subependymoma*.ab,ti,kf. \\
\hline 21 & ependymoblastoma*.ab,ti,kf. \\
\hline 22 & ganglioglioma*.ab,ti,kf. \\
\hline 23 & medulloblastoma*.ab,ti,kf. \\
\hline 24 & exp Germinoma/ \\
\hline 25 & germinoma*.ab,ti,kf. \\
\hline 26 & Meningioma/ \\
\hline 27 & meningioma*.ab,ti,kf. \\
\hline 28 & oligodendroglioma*.ab,ti,kf. \\
\hline 29 & exp Neurofibromatoses/ \\
\hline 30 & neurofibromatos*.ab,ti,kf. \\
\hline 31 & PNET $^{*} . a b, t i, k f$. \\
\hline 32 & neurocytoma*.ab,ti,kf. \\
\hline 33 & choroid plexus papilloma*.ab,ti,kf. \\
\hline 34 & $\begin{array}{l}\text { ((brain or central nervous system or CNS or brainstem or brain stem } \\
\text { or cerebel* or cerebr* or hypothalam* or ventric* or intracranial or } \\
\text { midline or choroid plexus or infratentorial or supratentorial or } \\
\text { neuroectoderm* or germ cell*) adj5 (tumo?r or neoplasm* or } \\
\left.\text { cancer }{ }^{*}\right) \text { ).ab,ti,kf. }\end{array}$ \\
\hline 35 & $\begin{array}{l}10 \text { or } 11 \text { or } 12 \text { or } 13 \text { or } 14 \text { or } 15 \text { or } 16 \text { or } 17 \text { or } 18 \text { or } 19 \text { or } 20 \text { or } 21 \\
\text { or } 22 \text { or } 23 \text { or } 24 \text { or } 25 \text { or } 26 \text { or } 27 \text { or } 28 \text { or } 29 \text { or } 30 \text { or } 31 \text { or } 32 \text { or } \\
33 \text { or } 34\end{array}$ \\
\hline 36 & exp Obesity/ \\
\hline 37 & obes*.ab,ti,kf. \\
\hline 38 & Overweight/ \\
\hline 39 & over weight.ab,ti,kf. \\
\hline 40 & overweight.ab,ti,kf. \\
\hline
\end{tabular}

Table 1 Search strategy for MEDLINE (Continued)

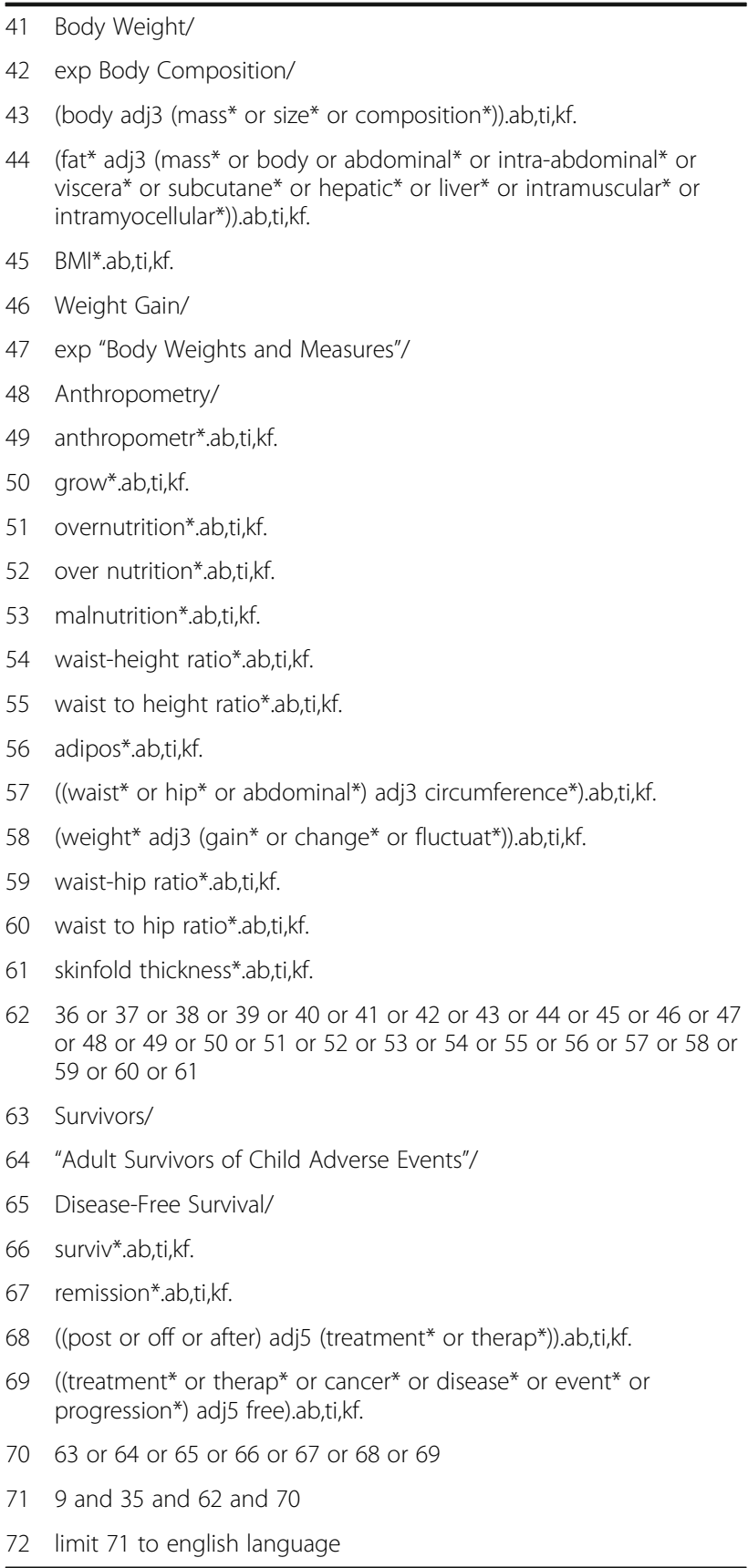

or BMI percentile. Secondary outcomes include waistto-hip ratio, waist-to-height ratio, body fat percentage, and skinfold thickness.

Risk of bias and quality assessment

Two reviewers will independently assess the risk of bias of the eligible studies using the Newcastle-Ottawa Scale (NOS) for observational studies [25]. The NOS will be adapted from its original version by considering a previously used modified version [26], so that the scale is 


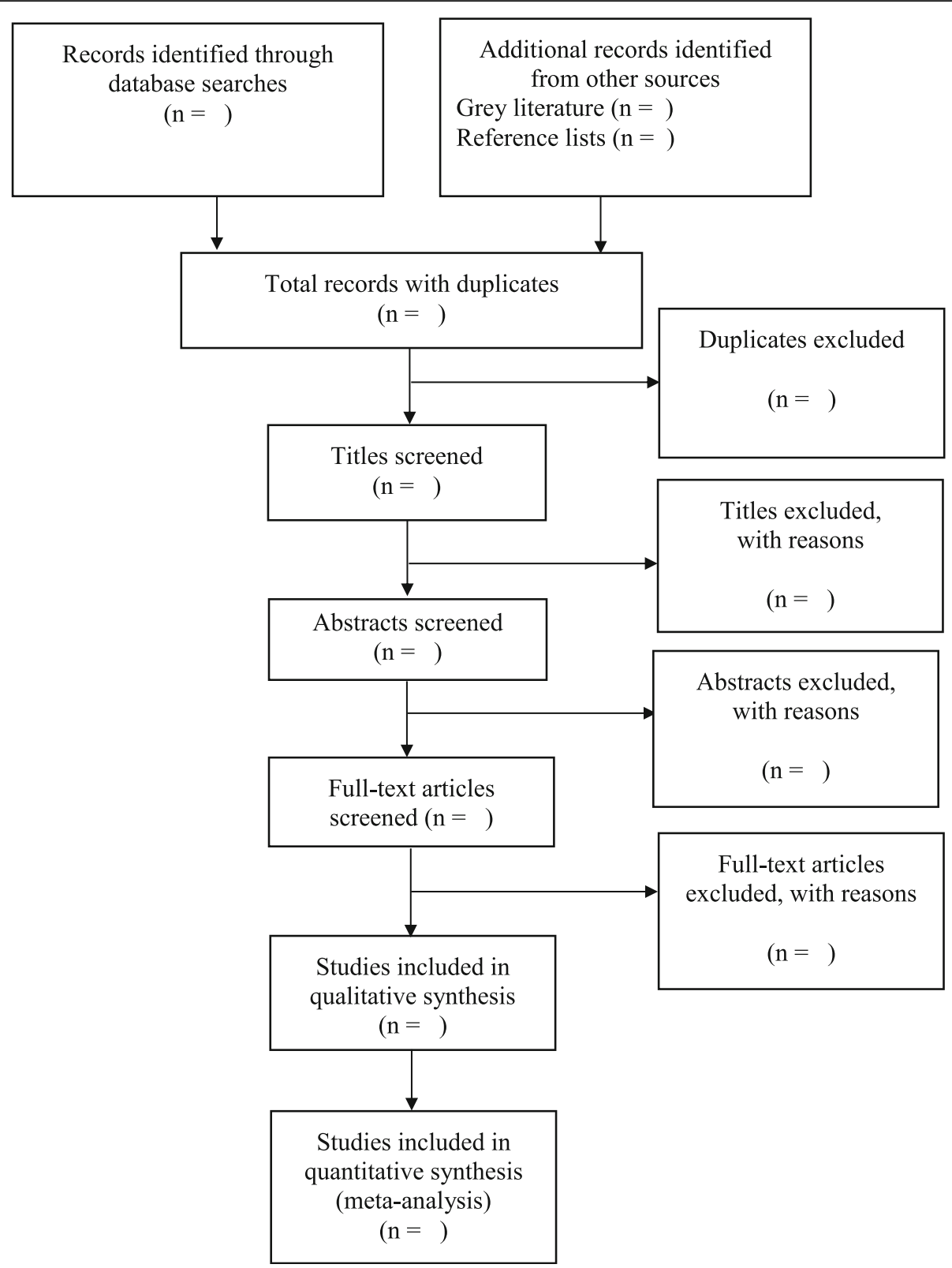

Fig. 1 PRISMA flow diagram

specific to this review. The reviewers will meet and discuss their decisions to include articles and to resolve any disagreement. In the case of persisting conflict, a third reviewer will be consulted.

This adapted NOS evaluates five items pertaining to risk of bias due to sample selection and classification (two items), confounding factors (one item), missing data (one item), and measurement errors (one item). For each item, the risk of bias is rated on a scale of 0 (high risk of bias), 1-2 (moderate risk of bias), and 3 (low risk of bias). The risk of bias is rated as unclear if not enough information is provided. Descriptions with examples for each level of risk of bias are provided (Additional file 2).
The overall risk of bias is rated as low when all five items have low risk of bias or high when one or more items have high risk of bias. The overall risk of bias is considered to be moderate when not all items have low risk of bias, but there are no items with high risk of bias. If one of the items is rated as unclear, the overall risk of bias will be reported as unclear as well.

Furthermore, we will use the Grading of Recommendations, Assessment, Development, and Evaluation (GRADE) guideline [27] to evaluate the overall quality of evidence including the risk of bias, inconsistency, indirectness, imprecision, and publication bias to determine the overall quality of evidence for each outcome. 


\section{Statistical analysis}

We will perform meta-analysis if two or more studies of similar design and population characteristics can be identified for each outcome. We expect high heterogeneity across studies. The possible sources of heterogeneity include age at diagnosis, duration and types of treatment, and brain tumor type and location. Therefore, we will perform meta-analysis using a random effects model if more than ten studies are eligible and will perform both random effects and fixed effects models if less than ten studies are identified [28].

Dichotomous and continuous outcomes will be reported as pooled odds ratio and standardized mean difference with 95\% confidence intervals, respectively. In studies where multiple measurements are done, we will include the outcomes measured with the longest follow-up reported.

Both inconsistency index $\left(I^{2}\right)$ and $P$ values from the chi-square test for homogeneity will be considered to determine the level of heterogeneity among the included studies. The threshold set by the Cochrane Collaboration will be used to interpret $I^{2}$, with $>75 \%$ representing considerable heterogeneity. A $P$ value of $<0.10$ will be used to determine statistical significance [29]. If meta-analysis is not appropriate, heterogeneity will be evaluated by describing and comparing the study samples, methods, and designs across studies. We will perform subgroup metaanalysis by sex and receipt of radiotherapy, chemotherapy, and surgery or combination therapies with these modalities if appropriate, as it has been reported that female SCBT are at higher risk of obesity than males $[7,8,11]$. In addition, to test the impact of outliers and studies with high risk of bias on the results, we will perform sensitivity analysis by excluding these studies if ten or more studies can be identified for an outcome.

To maintain the power of the results, we will not perform sensitivity analyses if less than ten studies are eligible. If ten or more studies are identified, we will use a contour-enhanced funnel plot to investigate publication bias [30]. The plot asymmetry will be determined by Egger's test and visual inspection [30]. Otherwise, we will estimate publication bias based on the number of relevant conference abstracts that did not have published articles originating from the work presented in the abstracts [31].

We will use Review Manager Version 5.3 Software (RevMan 5.3) [32] to conduct the meta-analysis. If Egger's test is appropriate, Comprehensive MetaAnalysis Software Version 3 (CMA 3.0) will be used instead [33]. A comprehensive table for summary of findings with narrative description will be reported when a meta-analysis is not appropriate.

We will report the results of this systematic review in accordance with the Preferred Reporting Items for Systematic Reviews and Meta-Analyses (PRISMA) guidelines using the PRISMA checklist [22, 23]. We will also document the date and reasons for any amendments to the protocol.

\section{Discussion}

While record numbers of children are surviving the diagnosis of brain tumors, this survival is burdened by the high rate of comorbidities and premature mortality $[10,12,34]$. To improve the quality of the cure, detailed understanding of the factors driving comorbidities in SCBT is likely to provide therapeutic entry points to improve outcomes.

Recent evidence suggests that new emerging risk factors may be contributing to mortality in this population. With increasing longevity, SCBT are at risk of type 2 diabetes and cardiovascular diseases that appear relatively early in life $[3-6,9]$. This argues for a premature aging process, whereby diseases of old age are appearing earlier in life in SCBT. This may indicate that similar overweight or obesity levels may have a disproportionately negative impact on SCBT when compared to the general population, and interventions are needed to stem the occurrence of overweight and obesity and reduce their burden in survivors. Notable limitations of this systematic review include the restriction of the search strategy to English language publications only, as this may lead to missing information from non-English literature. In addition, if the heterogeneity of the studies is high, this will preclude the performance of a metaanalysis. Nevertheless, this review will identify gaps in knowledge and inform better clinical practice in identifying overweight and obesity and will help inform the need for specifically designed interventions to tackle overweight and obesity in SCBT and improve outcomes.

\section{Additional files}

Additional file 1: PRISMA-P checklist. This checklist includes recommended items to address in a systematic reviews protocol and where they are reported in this protocol. (DOCX $36 \mathrm{~kb}$ )

Additional file 2: Adapted version of a modified Newcastle-Ottawa Scale (NOS) to evaluate overweight and obesity in survivors of childhood brain tumors. This form demonstrates the adapted version of the NOS to evaluate risk of bias of the included observational studies in this systematic review. (DOCX $17 \mathrm{~kb})$

\section{Abbreviations}

BMI: Body Mass Index; CINAHL: Cumulative Index to Nursing and Allied Health Literature; CMA 3.0: Comprehensive Meta-Analysis Software Version 3.0; EMBASE: Excerpta Medica dataBASE ; GRADE: Grading of Recommendations, Assessment, Development and Evaluation; MEDLINE: Medical Literature Analysis and Retrieval System Online; NOS: Newcastle-Ottawa Scale; PRISMA: Preferred Reporting Items for Systematic Reviews and Meta-Analyses; PRISMA-P: Preferred Reporting Items for Systematic Review and Meta-Analysis-Protocols; RevMan 5.3: Review Manager Version 5.3 Software; SCBT: Survivors of Childhood Brain Tumors 


\section{Acknowledgements}

We thank Ms. Pei-Wen Wang for editorial comments on this manuscript.

\section{Funding}

MCS was funded by the Hamilton Health Sciences and Foundation and the Pediatric Oncology Group of Ontario (POGO). KWW was funded by the Ontario Graduate Scholarship Program and the Canadian Institutes of Health Research (CIHR) Canada Graduate Scholarship-Masters. The funding agencies had no input into the design, conduct, or reporting of the data included in the systematic review.

\section{Availability of data and materials}

Not applicable.

\section{Authors' contributions}

MCS is the guarantor. Research question was defined by KWW, MCS, AF, SKS, RJdS, and LT. LB, KWW, RJdS, LT, and MCS contributed to the development of search strategy and determination of the eligibility criteria. Data abstraction form was designed by KWW and MCS. RJdS and LT provided the methodological support for this review. KWW and MCS drafted the manuscript, and the final version was reviewed and approved by all authors.

\section{Competing interests}

The authors declare that they have no competing interests.

\section{Consent for publication}

Not applicable.

\section{Ethics approval and consent to participate}

Not applicable.

\section{Author details}

'Department of Pediatrics, McMaster University, 1280 Main Street West, HSC-3A57, Hamilton, Ontario L8S 4K1, Canada. ${ }^{2}$ Division of Pediatric Endocrinology, McMaster Children's Hospital, Hamilton, Ontario, Canada. ${ }^{3}$ Medical Sciences Graduate Program, McMaster University, Hamilton, Ontario, Canada. ${ }^{4}$ Division of Pediatric Hematology/Oncology, McMaster Children's Hospital, Hamilton, Ontario, Canada. ${ }^{5}$ Division of Neurosurgery, Department of Surgery, McMaster Children's Hospital, Hamilton, Ontario, Canada. ${ }^{6}$ McMaster Stem Cell and Cancer Research Institute, McMaster University, Hamilton, Ontario, Canada. ${ }^{7}$ Health Sciences Library, McMaster University, Hamilton, Ontario, Canada. ${ }^{8}$ Department of Health Research Methods, Evidence and Impact, McMaster University, Hamilton, Ontario, Canada. ${ }^{9}$ Department of Anesthesia, McMaster University, Hamilton, Ontario, Canada. ${ }^{10} \mathrm{Centre}$ for Evaluation of Medicines, St. Joseph's Health Care, Hamilton, Ontario, Canada. ${ }^{11}$ Biostatistics Unit, St Joseph's Healthcare, Hamilton, Ontario, Canada.

\section{Received: 21 December 2016 Accepted: 20 February 2017}

\section{Published online: 03 March 2017}

\section{References}

1. Miller KD, Siegel RL, Lin CC, Mariotto AB, Kramer JL, Rowland JH, et al. Cancer treatment and survivorship statistics, 2016. CA Cancer J Clin. 2016; 66(4):271-89.

2. Woehrer A, Hackl M, Waldhor T, Weis S, Pichler J, Olschowski A, et al. Relative survival of patients with non-malignant central nervous system tumours: a descriptive study by the Austrian Brain Tumour Registry. Br J Cancer 2014:110(2):286-96.

3. Chambless LB, Parker SL, Hassam-Malani L, McGirt MJ, Thompson RC. Type 2 diabetes mellitus and obesity are independent risk factors for poor outcome in patients with high-grade glioma. J Neurooncol. 2012;106(2):383-9.

4. Gurney JG, Kadan-Lottick NS, Packer RJ, Neglia JP, Sklar CA, Punyko JA, et al. Endocrine and cardiovascular late effects among adult survivors of childhood brain tumors: Childhood Cancer Survivor Study. Cancer. 2003;97(3):663-73.

5. Heikens J, Ubbink MC, van der Pal HP, Bakker PJ, Fliers E, Smilde TJ, et al, Long term survivors of childhood brain cancer have an increased risk for cardiovascular disease. Cancer. 2000;88(9):2116-21.

6. Holmqvist AS, Olsen JH, Andersen KK, de Fine LS, Hjorth L, Garwicz S, et al. Adult life after childhood cancer in Scandinavia: diabetes mellitus following treatment for cancer in childhood. Eur J Cancer. 2014;50(6):1169-75.
7. Lek N, Prentice P, Williams RM, Ong KK, Burke GA, Acerini CL. Risk factors for obesity in childhood survivors of suprasellar brain tumours: a retrospective study. Acta Paediatr. 2010;99(10):1522-6.

8. Lustig RH, Post SR, Srivannaboon K, Rose SR, Danish RK, Burghen GA, et al. Risk factors for the development of obesity in children surviving brain tumors. J Clin Endocrinol Metab. 2003:88(2):611-6.

9. Meacham LR, Sklar CA, Li S, Liu Q, Gimpel N, Yasui Y, et al. Diabetes mellitus in long-term survivors of childhood cancer. Increased risk associated with radiation therapy: a report for the Childhood Cancer Survivor Study. Arch Intern Med. 2009;169(15):1381-8.

10. Mertens AC, Yasui Y, Neglia JP, Potter JD, Nesbit Jr ME, Ruccione K, et al. Late mortality experience in five-year survivors of childhood and adolescent cancer: the Childhood Cancer Survivor Study. J Clin Oncol. 2001;19(13):3163-72.

11. Pietilä S, Mäkipernaa A, Sievänen H, Koivisto AM, Wigren T, Lenko HL. Obesity and metabolic changes are common in young childhood brain tumor survivors. Pediatr Blood Cancer. 2009;52(7):853-9.

12. Prasad PK, Signorello LB, Friedman DL, Boice Jr JD, Pukkala E. Long-term non-cancer mortality in pediatric and young adult cancer survivors in Finland. Pediatr Blood Cancer. 2012;58(3):421-7.

13. Poirier P, Giles TD, Bray GA, Hong Y, Stern JS, Pi-Sunyer FX, et al. Obesity and cardiovascular disease: pathophysiology, evaluation, and effect of weight loss. Arterioscler Thromb Vasc Biol. 2006;26(5):968-76.

14. Green DM, Cox CL, Zhu L, Krull KR, Srivastava DK, Stovall M, et al. Risk factors for obesity in adult survivors of childhood cancer: a report from the Childhood Cancer Survivor Study. J Clin Oncol. 2012;30(3):246-55.

15. Mulrooney DA, Ness KK, Neglia JP, Whitton JA, Green DM, Zeltzer LK, et al. Fatigue and sleep disturbance in adult survivors of childhood cancer: a report from the Childhood Cancer Survivor Study (CCSS). Sleep. 2008: 31(2):271-81

16. Ness KK, Morris EB, Nolan VG, Howell CR, Gilchrist LS, Stovall M, et al. Physical performance limitations among adult survivors of childhood brain tumors. Cancer. 2010;116(12):3034-44.

17. Oberfield SE, Sklar CA. Endocrine sequelae in survivors of childhood cancer. Adolesc Med. 2002;13(1):161-9. viii.

18. Moher D, Shamseer L, Clarke M, Ghersi D, Liberati A, Petticrew M, et al. Preferred Reporting Items for Systematic Review and Meta-analysis Protocols (PRISMA-P) 2015 statement. Syst Rev. 2015;4:1.

19. Moher D, Stewart L, Shekelle P. Implementing PRISMA-P: recommendations for prospective authors. Syst Rev. 2016;5(1):15.

20. EndNote [Computer program]. Version 7.7.1. Clarivate Analytics; 2016.

21. Zhang FF, Liu S, Chung M, Kelly MJ. Growth patterns during and after treatment in patients with pediatric ALL: a meta-analysis. Pediatr Blood Cancer. 2015;62(8):1452-60.

22. Liberati A, Altman DG, Tetzlaff J, Mulrow C, Gotzsche PC, loannidis JP, et al. The PRISMA statement for reporting systematic reviews and meta-analyses of studies that evaluate healthcare interventions: explanation and elaboration. BMJ. 2009:339:b2700

23. Moher D, Liberati A, Tetzlaff J, Altman DG, PRISMA Group. Preferred Reporting Items for Systematic Reviews and Meta-Analyses: the PRISMA statement. Int J Surg. 2010;8(5):336-41.

24. Wang K-W, Valencia M, Banfield L, Chau R, Fleming A, Singh SK, et al. The effectiveness of interventions to treat obesity in survivors of childhood brain tumors: a systematic review protocol. Syst Rev. 2016:5:101.

25. Wells GA, Shea B, O'Connell D, Peterson J, Welch V, Losos M, et al. The Newcastle-Ottawa Scale (NOS) for assessing the quality if nonrandomized studies in meta-analyses. 2009. http://www.ohri.ca/programs/clinical_ epidemiology/oxford.asp. Accessed 15 Nov 2016.

26. Bawor M, Dennis BB, Anglin R, Steiner M, Thabane L, Samaan Z. Sex differences in outcomes of methadone maintenance treatment for opioid addiction: a systematic review protocol. Syst Rev. 2014;3:45.

27. Atkins D, Best D, Briss PA, Eccles M, Falck-Ytter Y, Flottorp S, et al. Grading quality of evidence and strength of recommendations. BMJ. 2004; 328(7454):1490.

28. Borenstein M, Hedges LV, Higgins JP, Rothstein HR. A basic introduction to fixed-effect and random-effects models for meta-analysis. Res Synth Methods. 2010;1(2):97-111.

29. Deeks JJ, Higgins JPT, Altman DG (editors). Chapter 9: Analysing data and undertaking meta-analyses. In: Higgins JPT, Green S, editors. Cochrane handbook for systematic reviews of interventions version 5.1.0. (updated March 2011). The Cochrane Collaboration; 2011. Available from www. handbook.cochrane.org. 
30. Sterne JAC, Egger M, Moher D (editors). Chapter 10: Addressing reporting biases. In: Higgins JPT, Green S (editors). Cochrane handbook for systematic reviews of intervention version 5.1.0. (updated March 2011). The Cochrane Collaboration; 2011. Available from www.handbook.cochrane.org.

31. Shakiba S, Shakiba B, Irani S. Unpublished abstracts can be invaluable. Can Urol Assoc J. 2014;8(1-2):E60.

32. Review Manager (RevMan) [Computer program]. Version 5.3. Copenhagen: The Nordic Cochrane Centre, The Cochrane Collaboration; 2014.

33. Comprehensive Meta-Analysis (CMA) [Computer program]. Version 3. https://www.meta-analysis.com/.

34. Oeffinger KC, Mertens AC, Sklar CA, Kawashima T, Hudson MM, Meadows AT, et al. Chronic health conditions in adult survivors of childhood cancer. N Engl J Med. 2006;355(15):1572-82.

Submit your next manuscript to BioMed Central and we will help you at every step:

- We accept pre-submission inquiries

- Our selector tool helps you to find the most relevant journal

- We provide round the clock customer support

- Convenient online submission

- Thorough peer review

- Inclusion in PubMed and all major indexing services

- Maximum visibility for your research

Submit your manuscript at www.biomedcentral.com/submit
Biomed Central 